Bangladesh J. Bot. 42(1): 23-29, 2013 (June)

\title{
LOCATION AND CHEMICAL COMPOSITION OF SEMI-PERMEABLE LAYER OF FORAGE SEEDS
}

\author{
Jing Zhou, YANRONG WANG* AND Zulfi JAHUfER ${ }^{1}$ \\ State Key Laboratory of Grassland Agro-ecosystems, College of Pastoral Agriculture Science and \\ Technology, Lanzhou University, P.O. Box 61, Lanzhou 730020, China
}

Key words: Seeds, Semi-permeable layer, Location, Chemical composition

\begin{abstract}
The presence of the semi-permeable layer is determined in Roegneria nutans (Keng.) Keng, Achnatherum inebrians (Hance.) Keng, Hordeum vulgare var. nudum Hook. f., Triticale, Festuca sinensis Keng., and Bromus inermis Leyss. using the lanthanum nitrate tracer method, transmission electron microscopy and energy dispersive X-ray analysis. It was an amorphous membranous structure firmly attached on the external portion of the seed coat. The diversified chemical composition of the semi-permeable layer of the species studied is also analyzed by histochemical staining. $R$. nutans, A. inebrians, $H$. vulgare var. nudum, and Triticale were found to contain lipids in the semi-permeable layer. F. sinensis had pectin whereas $B$. inermis has cellulose in the said layer.
\end{abstract}

\section{Introduction}

The semi-permeable layer is an amorphous tissue that covers the seed and restricts or impedes solute exchange while allowing the permeability of internal and external water and gas (Beresniewicz et al. 1995a). Studies have shown that this layer can prevent the absorption of toxic substances by seeds in hazardous environments (Wolfe 1926). A water film can be formed around the embryo by this layer, through which water can be stored to protect the mature embryo from drought stress (Prutsch et al. 2000). Therefore, the semi-permeable layer is valuable protection to sustain the health and secure the growth, development, germination, and quality testing of seeds (Salanenka et al. 2009, Zhou and Wang 2012).

The location, chemical composition and function of the semi-permeable layer has been investigated widely in a number of plants (Hill and Taylor 1989, Salanenka and Taylor 2009, Yim and Bradford 1998, Ramakrishna and Amritphale 2005).

Previous studies on the semi-permeable layer mainly involved the seeds of fruits, vegetables, and crops. Those studies that investigated forage seeds were only reports on the location of this layer in Sudan grass, Elymus sibiricus L., Lolium perenne L., Elymus nutans Griseb. (Yan 2008), and Leymus chinensis (Trin.) Tzvel (He 2011). Few plant species have been studied specifically for this layer, and the research done is insufficient. In the present paper, six kinds of excellent forage seeds, including Roegneria nutans (Keng.) Keng, Achnatherum inebrians (Hance.) Keng, Hordeum vulgare var. nudum Hook. f., Triticale, Festuca sinensis Keng., and Bromus inermis Leyss. were investigated. Among these plants, the R. nutans, A. inebrians, H. vulgare var. nudum, and $F$. sinensis are dominant or constructive species in the grasslands of the Gannan Prairie in Gansu, China. The Triticale is a highly economically valuable forage crop in the Northwest Loess Plateau (He et al. 2012). The B. inermis is an important forage seed with strong cold resistance and widely distributed in the pasture meadows of the mountainous areas in northern China (Casler and Brummer 2005, Zhang et al. 2011). The present study was undertaken to determine the position of the semi-permeable layer in the six forage seeds.

"Author for correspondence: <yrwang2007@yahoo.cn>. ${ }^{1}$ Agresearch, Grasslands Research Centre, Private Bag 11008, Palmerston North, New Zealand. 


\section{Materials and Methods}

Seed samples of $R$. nutans, A. inebrians, $H$. vulgare var. nudum and $F$. sinensis were collected from the Gannan Prairie in Gansu, China (102 $31^{\prime}$ E and $\left.35^{\circ} 12^{\prime} \mathrm{N}\right)$, in September 2009. The Triticale was obtained from the Seed Management Station in Gansu, China, whereas $B$. inermis was provided by the Official Herbage and Turfgrass Seed Testing Centre of the Ministry of Agriculture in Lanzhou, China. The palea and lemma were removed from the seeds, and the 'naked' seed was then fixed at $4^{\circ} \mathrm{C}$ for $24 \mathrm{~h}$ in $4 \%$ glutaraldehyde in phosphate buffer $(25 \mathrm{mM}, \mathrm{pH}$ 6.8) for observation by light microscopy. The remaining seeds were placed at room temperature and dried naturally after collection for preservation.

For the transmission electron micrograph (TEM) analysis, the lanthanum nitrate tracer method was followed (Beresniewicz et al. 1995a, Zhu et al. 2009). Thirty seeds were collected for each sample, stripped of the inside and outside lemma and soaked in double-distilled water at $20^{\circ} \mathrm{C}$ for $24 \mathrm{~h}$ incubation. The seeds were observed under an illuminated stereomicroscope (Leica ZOOM 2000 , USA) to check for intact seeds. Those with surface breakage were removed and the remaining seeds were soaked in $2 \%$ lanthanum nitrate solution, prepared with double-distilled water to remove $\mathrm{CO}_{2}$, for $24 \mathrm{~h}$. The surfaces of the seeds were cut with a razor blade to remove approximately $1 \mathrm{~mm}^{3}$ of the caryopsis coat with a small amount of endosperm tissue. The cut out sections were then fixed in $4 \%$ glutaraldehyde solution for $24 \mathrm{~h}$ and ultrathin sections of the seed were prepared after Zheng (1993). The sections were stained with lead citrate and uranyl acetate and were observed and photographed under TEM (JEOL-1230, Japan). The semi-permeable layer was identified as the internal structure of the seed if it prevented the penetration of lanthanum.

EDX: It was performed following the same way as with TEM. The seeds soaked and integrated were drawn out and aired at room temperature after $24 \mathrm{~h}$ incubation in lanthanum nitrate solution. The seeds were then cut longitudinally with a razor blade and mounted on an aluminum shell. The seed structure was viewed with a scanning electron microscope (SEM) (JSM 5600 LV, Japan) (Engin 2008). The seeds were sputter-coated with gold and viewed with the SEM in backscattered electron imaging mode at $20 \mathrm{kV}$. Salt ions identified in the seed samples were analyzed for $20 \mathrm{sec}$. The lanthanum concentrations used were based on the L-series.

Light microscopy and histochemical analysis: The fixed samples were rinsed with PBS $(0.1 \mathrm{~mol} / \mathrm{l}, \mathrm{pH} 7.2)$ three times for $10 \mathrm{~min}$ in each time and dehydrated by alcohol gradient, once with $15,30,50,70$ and $95 \%$ and twice with $100 \%$. The samples were pre-impregnated with $1 / 2$ Technovit 7100 and $1 / 2100 \%$ ethanol for over $3 \mathrm{~h}$ and soaked in Technovit 7100 and Hardener I at a ratio of $100: 1$ for $24 \mathrm{~h}$. The samples were embedded and polymerized with Technovit 7100 embedding agent and cut into $2-4 \mu \mathrm{m}$ thick slices with the semi-thin slices machine (KD202B, China). The sections were stained with $0.05 \%$ aniline blue to determine their microscopic anatomical structures. The sections were stained for about 10 min with $0.2 \%$ Sudan III prepared with $70 \%$ alcohol as previously described (Yim and Bradford 1998). According to the method previously described by $\mathrm{Li}$ (1996), the slices were stained with saturated ruthenium red for about $30 \mathrm{~min}$. After staining, the samples were washed thoroughly in water and covered with the slide. Then two to three drops of iodine solution were added to the slices and allowed to soak through for $10 \mathrm{~s}$, after which, a few drops of saturated lithium chloride solution were added for observation after covering the slide. All samples were observed under a compound microscope (Nikon Eclipse E 100, Japan) and photographed.

\section{Results and Discussion}

The semi-permeable layer is presumably located at the position where the lanthanum is prevented from penetrating. As shown in Fig. A1-C1 and Fig. 2A1-C1, abundant lanthanum 
deposits are found on the pericarps (shown by the black arrow) of R. nutans, A. inebrians, $H$. vulgare var. nudum, Triticale, $F$. sinensis, and $B$. inermis, blocked by a layer of transparent, amorphous membranous structure (shown by*). Observation of the inner parts of the seeds shows that no flocculent crystalline materials (shown by the white arrow) were found on the thickened periclinal wall of the layer of aleurone cells in R. nutans (Fig. 1A2), thus proving that the barrier preventing the permeation of lanthanum is the semi-permeable layer. Observation of the seed coat and aleurone layer structure (Fig. 1B2) of A. inebrians reveals that no flocculent crystals were present on the cell wall, proving that the lanthanum has not infiltrated into the seed coat. Similar to in the observation for $R$. nutans, the lanthanum was blocked in the outermost coats of the $A$. inebrians seeds. The microstructure of the H. vulgare var. nudum seed coat and thickened periclinal wall of the aleurone cells is illustrated in Fig. 1C2, which indicates no lanthanum particles (white arrows) in either of the two seed structures inspected, indicating that the lanthanum was blocked outside the seed coat.
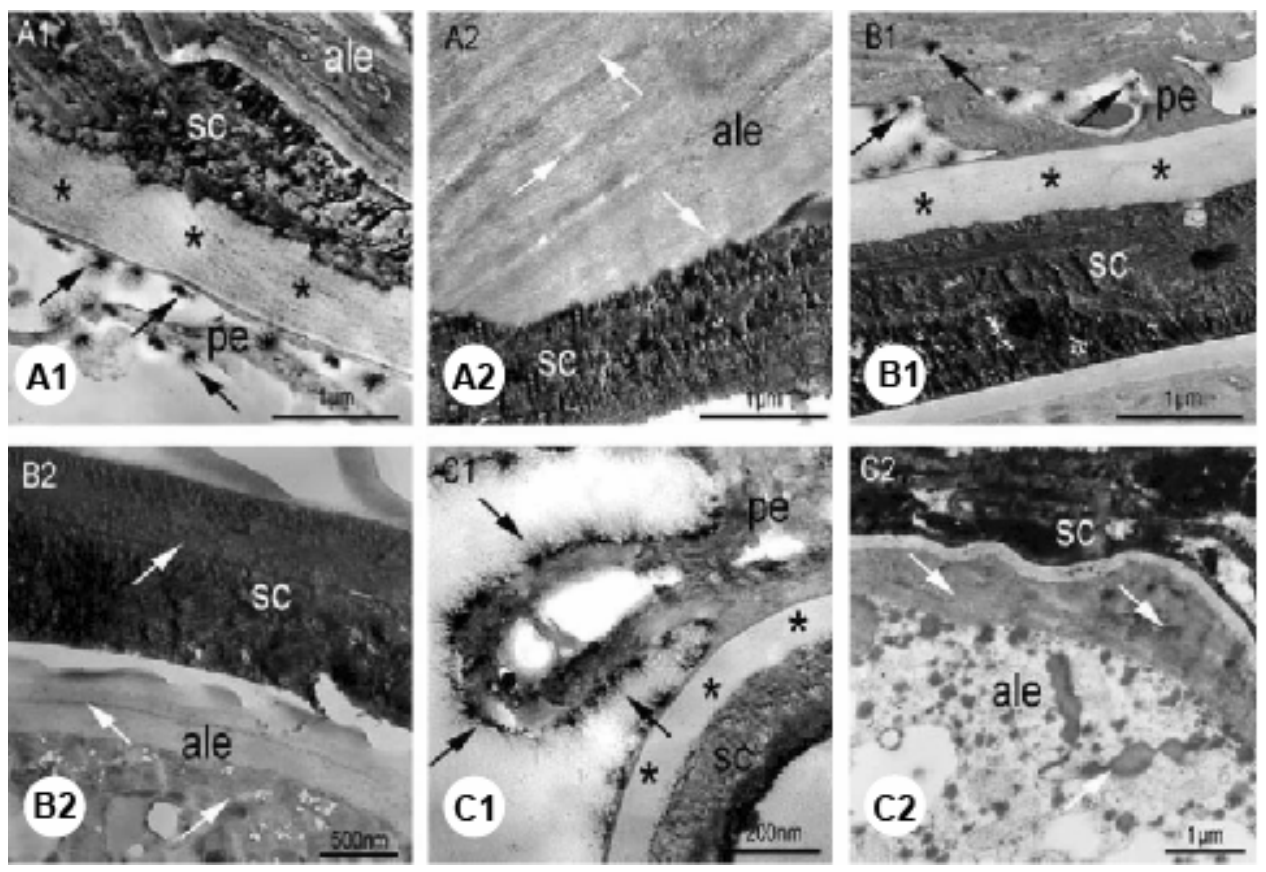

Fig. 1A-C. TEM images of the semi-permeable layer of (A) Roegneria nutans, (B) Achnatherum inebrians, and (C) Hordeum vulgare var. nudum. 1: location of the seed pericarp (pe), with abundant lanthanum like flocculent structure. ${ }^{*} \mathrm{C}$ represents the semi-permeable layer. 2: seed coat (sc) and the thickened wall of the aleurone cell (ale). Black and white arrows indicate the presence and absence of lanthanum, respectively

Observations of the caryopsis coat microstructures of Triticale, F. sinensis and B. inermis (Fig. 2A2 - C2) reveal that the lanthanum was not deposited on the seed coat and aleurone cells (white arrows), thus indicating the lanthanum was unable to seep into the structure of the seed coat. The thick, film-like layer external to it is the semi-permeable layer, which can be seen closely connected with the seed coat and deemed responsible for blocking lanthanum. Under TEM, a semi-permeable layer was observed preventing the penetration of lanthanum in all six forage seed types. 
Further evidence from elemental analysis shows that the flocculent crystals are lanthanum, which is found in significantly high levels in the pericarp of the forage seeds. The absence of lanthanum peaks in the seed coat confirms that the semi-permeable layer of the six forage seeds is located between the pericarp and the seed coat. TEM further indicated that the semi-permeable layer, which can be easily distinguished from the pericarp and seed coat structure, is a transparent, amorphous, and membranous structure firmly attached to the seed coat.
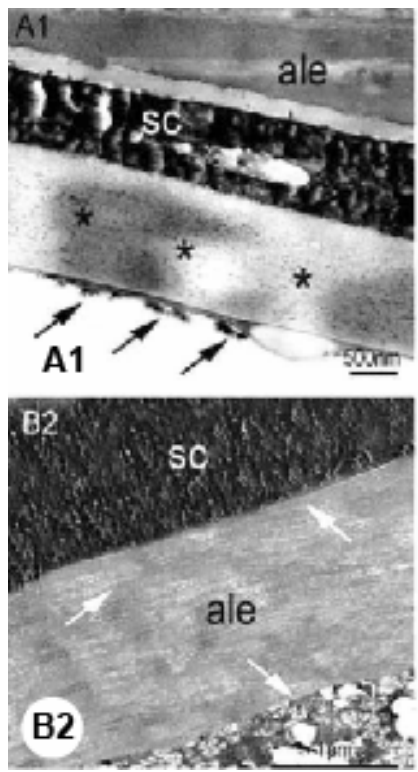
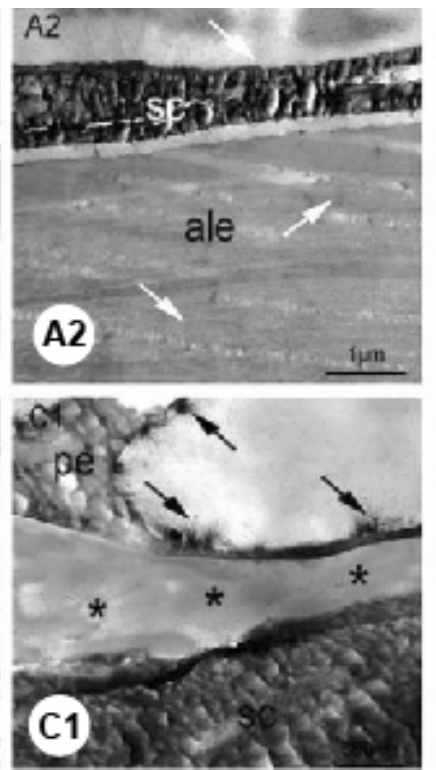

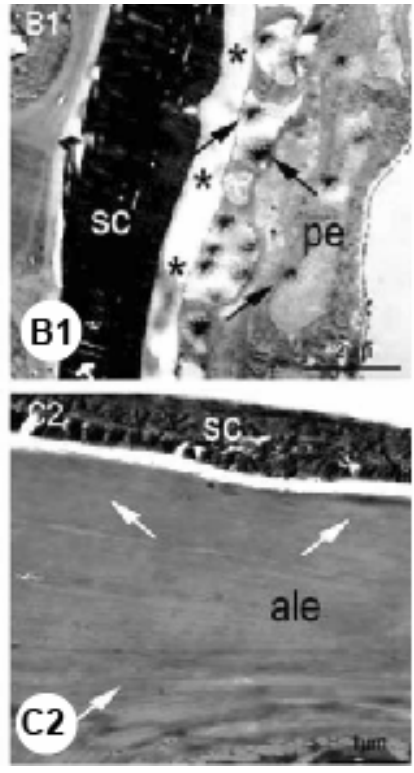

Fig. 2A-C. TEM images of the semi-permeable layer of (A) Triticale, (B) Festuca sinensis, and (C) Bromus inermis. 1: location of the seed pericarp (pe) with abundant lanthanum like flocculent structure. *represents the semi-permeable layer. 2: seed coat (sc) and thickened wall of the aleurone cell (ale). Black and white arrows indicate the presence and absence of lanthanum, respectively.

Chemical composition of the semi-permeable layer of seeds: The anatomical structures of the pericarps, the semi-permeable layers, seed coats, and aleurone layers of $R$. nutans, $A$. inebrians, $H$. vulgare var. nudum, Triticale, F. sinensis, and B. inermis are illustrated in Fig. 3 . The semipermeable layer is located external to the seed coat and tightly attached to it. Histochemical analysis shows that this layer in $R$. nutans, A. inebrians, $H$. vulgare var. nudum, and Triticale were stained red with $0.2 \%$ Sudan III, indicating that a major component of the chemical composition of this layer is lipid (Fig. 3A-D). However, the semi-permeable layer of F. sinensis and B. inermis were not stained by Sudan III. For the six forage seeds studied, this layer was also stained with ruthenium red solution. Only the semi-permeable layer of $F$. sinensis was stained red, indicating that the main chemical component of the layer is pectin (Fig. 3E). The semi-permeable layer of $B$. inermis was dyed yellow by the iodine solution and saturated lithium chloride solution, indicating that the primary chemical component of this layer is cellulose (Fig. 3F).

In this research, the structure of the caryopsis coat of the six forage seeds were studied using the lanthanum tracer, TEM, and EDX techniques. The results show the existence of a semipermeable layer in the seeds, firmly attached and located external to the seed coat (Figs 1 - 2). In the study of the seed coat structure of switchgrass (Panicum virgatum L.), Salanenka and Taylor (2009) found the structure prevented the penetration of fluorescent dye, meaning the semi- 
permeable layer of the plant is located in the seed coat. Leek, onion, tomato, and pepper all have a semi-permeable layer on the inner section of the seed coat, close to the endosperm tissue (Beresniewicz 1995a,b). Hill and Taylor (1989) maintained that lettuce seeds have an endospermic semi-permeable layer around the embryo, forming a permeable barrier organization. The endosperm tissue of castor seeds also prevents the penetration of fluorescent dye, indicating that the semi-permeable layer of the seed is located on the endosperm (Salanenka and Taylor 2009). Studies on Sudan grass seeds show that the semi-permeable layer is located on the inner side of the aleurone layer, close to the undifferentiated cells, and is, hence, endospermic (Yan 2008). Thus, the location of the semi-permeable layer varies with the plant species. However, regardless of the location, the function is the same, which is preventing or restricting material exchange between the internal and the external environments of the seed. Furthermore, TEM observations in the current study show that the semi-permeable layer of the six forage seeds, which is a transparent amorphous membranous structure, can be easily distinguished from the pericarp and seed coat structure (Figs 1 - 2). This finding is consistent with those of Beresniewicz et al. (1995a) in their study of the semi-permeable layer in onion, leek, pepper, and tomato.
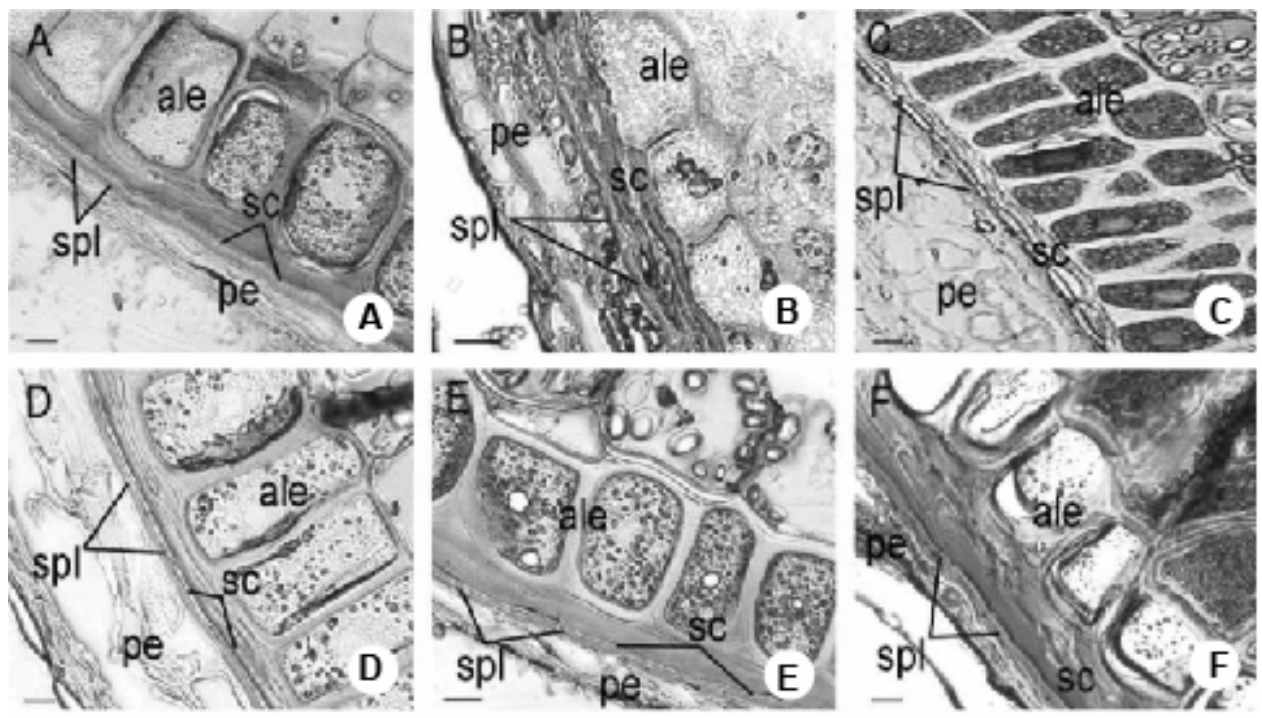

Fig. 3A-F. Anatomical structures of the pericarp (pe), seed coat (sc), and aleurone cell (ale) of the seed and the chemical nature of the semi-permeable layer (spl). A-D: The semi-permeable line of Roegneria nutans, Achnatherum inebrians, Hordeum vulgare var. nudum, and Triticale, which can be stained by the Sudan III, respectively. E and F: Festuca sinensis and Bromus inermis stained red and yellow with ruthenium red and iodine and lithium chloride solution, respectively (Bars: $10 \mu \mathrm{m}$ ).

The characteristics of the semi-permeable layer are assumed closely related to the chemical substances deposited around it (Beresniewicz et al. 1995b). Thus, the primary chemical component of the semi-permeable layer of leek and onion seed is cutin, whereas that of tomato and pepper is suberin. Given the permeability of leek and onion is higher than that of tomato and pepper, the inhibiting feature of suberin is presumed more efficient than that of cutin (Beresniewicz et al. 1995b). Yim and Bradford (1998) found that cucumber and watermelon seeds have a semi-permeable layer primarily composed of callose, not cutin or suberin, as previously believed (Yim and Bradford 1998). However, no uniform conclusion has been reached on the chemical composition of this layer in muskmelon, cucumber, and watermelon seeds. It is assumed 
that the semi-permeable characteristics of the layer are attributed to the high lipid content in these plants (Ramakrishna and Amritphale 2005). The present study reveals that chemical composition of this layer in R. nutans, A. inebrians, H. vulgare var. nudum and Triticale is lipid (Fig. 3A-D), whereas those of $F$. sinensis and B. inermis is pectin and cellulose, respectively (Fig. 3E-F). Thus, the chemical composition of the semi-permeable layer not only determines the permeability of the layer, but also shows diversity across species. Therefore, the study of the chemical composition of the semi-permeable layer is of significant importance to understand its characteristics.

Overall, the species studied all have a semi-permeable layer located on the outer portion of the seed coat. The chemical nature of the semi-permeable layer varies with the species. The primary component in the semi-permeable layer of $R$. nutans, A. inebrians, $H$. vulgare var. nudum, and Triticale is lipid, whereas in those of $F$. sinensis and $B$. inermis, the primary component is pectin and cellulose, respectively.

\section{Acknowledgements}

Authors are grateful for the financial support provided by the Natural Science Foundation of China (Grant no. 30771532) and the National Basic Research Program (973 program) of China (grant no. 2007CB108904). They also thank Seeds Management Station in Gansu, China and Official Herbage and Turfgrass Seed Testing Centre of the Ministry of Agriculture in Lanzhou, China for providing seeds.

\section{References}

Beresniewicz MM, Taylor AG, Goffinet MC and Terhune BT 1995a. Characterization and location of a semipermeable layer in seed coats of leek and onion (Liliaceae), tomato and pepper (Solanaceae). Seed Sci. Technol. 23(1): 123-134.

Beresniewicz MM, Taylor AG, Goffinet MC and Koeller WD 1995b. Chemical nature of a semipermeable layer in seed coats of leek, onion (Liliaceae), tomato and pepper (Solanaceae). Seed Sci. Technol. 23(1): 135-145.

Casler MD and Brummer EC 2005. Forage yield of smooth bromegrass collections from rural cemeteries. Crop Sci. 45(6): 2510- 2516.

Engin H 2008. Scanning electron microscopy of floral initiation and developmental stages in Sweet cherry (Prunus avium L.) under water deficits. Bangladesh J. Bot. 37(1): 15-19.

He JF, Zhao ML, Zheng YH and Li M 2012. Forage characteristics and application perspective of Triticale in grassland ecology. Chinese J. Grassland 34(1): 101-107.

He XQ 2011. Seed coat permeability and location of semipermeable layer in seeds of several grass species. Lanzhou Univ. Dissertation, Lanzhou. pp. 2-3.

Hill HJ and Taylor AG 1989. Relationship between viability, endosperm integrity and imbibed lettuce seed density and leakage. HortScience 24(5): 814-816.

Li ZL 1996. Slice production science in plant tissue. Peking Univ. Press, Beijing. pp. 112-113.

Prutsch J, Schardt A and Schill R 2000. Adaptations of an orchid seed to water uptake and storage. Plant Syst. Evol. 220(1-2): 69-75.

Ramakrishna P and D Amritphale 2005. The perisperm-endosperm envelope in Cucumis: structure, proton diffusion and cell wall hydrolysing activity. Ann. Bot. 96(5): 769-778.

Salanenka YA, Goffinet MC and Taylor AG 2009. Structure and histochemistry of the micropylar and chalazal regions of the perisperm-endosperm envelope of cucumber seeds associated with solute permeability and germination. J. Amer. Soc. Hort. Sci. 134(4): 479-487.

Wolfe HS 1926. Absorption of water by barley seeds. Bot. Gaz. 82(1): 89-103.

Yan XJ 2008. Study on microstructure and location of semi-permeable layer in several grass species seed. Lanzhou Univ. Dissertation, Lanzhou. 
Yim KO and Bradford KJ 1998. Callose deposition is responsible for apoplastic semipermeability of the endosperm envelope of muskmelon seeds. Plant Physiol. 118(1): 83-90.

Zhang FX, Wang TJ, Zhao ZL, Du JC, Tian QS and Wu XH 2011. Genetic diversity analysis of 12 Bromus inermis Leyss populations based on RAPD markers. Chinese J. Grassland 33(2): 25-30.

Zheng GC 1993. Biological Microscopy Techniques. People's Education Press, Beijing. pp. 43-78.

Zhou J and Wang YR 2012. Research advances in semi-permeable layer of seed. Acta Bot. Boreal.-Occident. Sin. 32(9): 1928-1934.

Zhu QH, Zhang XC and Arunakumara KKIU 2009. Ultrastructure of sporophyte of Cladosiphon okamuranus Tokida (Ectocarpales, Phaeophyceae). Bangladesh J. Bot. 38(2): 177-180.

(Manuscript received on 8 November, 2012; revised on 7 April, 2013) 